\title{
Using Shack-Hartmann wavefront sensors and Zernike coefficients for beam characterisation: numerical procedures.
}

\author{
Salvador Bosch*a, Santiago Vallmitjana ${ }^{\mathrm{a}}$, Antonio Marzoa ${ }^{\mathrm{a}}$, Justo Arines ${ }^{\mathrm{b}}$, Eva Acosta $^{\mathrm{b}}$ \\ ${ }^{a}$ Dep. Física Aplicada, Universitat de Barcelona, Martí i Franques 1, 08028 Barcelona. Spain. \\ ${ }^{\mathrm{b}}$ Dep. Física Aplicada, Universidade de Santiago de Compostela, Facultad de Física. Spain.
}

\begin{abstract}
When using Shack-Hartmann wavefront sensors ( $\mathrm{SH}$ ) and Zernike coefficients (Zs) in applications where the position of the measurement and the point of interest are far apart, as it is common practice in ophthalmic optics, problems in the interpretation of the values of the Zs arise, related to how the shape of the wavefront propagates along the beam. One typical example is pupil conjugation where an auxiliary lens is added to match the size of the area of the interest of the beam with the size of the entrance pupil of the SH used for measurements.

In the present work, we address this problem in the framework of a numerical scheme for modeling the beam propagation. We calculate the wavefronts with exact ray tracing plus the fitting of the impacts so as to match a rectangular grid. This procedure allows the subsequent calculation of the Zs or, similarly, the pupil function at an arbitrary plane perpendicular to the optical axis. All the numerical methods and procedures have been implemented in MATLAB code and can be illustrated by running the MATLAB script for the setup configuration that is being considered. Several examples are presented to illustrate the previous ideas and to show the real capabilities of our procedures. They will help to clarify the issues actually found in practical setups for beam manipulation, often encountered in ophthalmic optics.
\end{abstract}

Keywords: Wavefront sensors, beam propagation, beam characterization, ophthalmic optics

\section{INTRODUCTION}

We want to investigate the potential use of a SH sensor for the characterization of light beams in optical setups. One of the main limitations for the utility of the SH sensor in that task may be due to the complicated shape of the wavefront at the testing point, for example, due to the crossing of light rays in the vicinity. In effect, if more than one ray arrives at a single point in the measurement zone, the slope of the wavefront (the quantity measured by the SH) is not a well-defined quantity and the usual computation methods devised for SH sensors collapse. ${ }^{1-4}$

To get a clear example to illustrate the above ideas, we will study in detail the pass of the light trough a meniscus lens, up to a several planes perpendicular to the optical axis. To have a general enough case, the object point is off axis. By analyzing in detail the optical paths and the shapes of the wavefronts, we will understand why the use of a SH at different positions of the beam path may lead or not to meaningful conclusions. We consider that this kind of information is very important in situations where pupil conjugation is achieved by using just one lens, or after passing through a beam diameter compressor built with a Galilean telescope. These configurations can be found quite often, as for example in the characterization of ophthalmic lenses or in beam characterization in ultra intense lasers.

The numerical procedures have been developed in MATLAB environment ${ }^{5}$, but many features have also been checked with $\mathrm{ZEMAX}^{6}$. In Section 2 we will describe the numerical tool we have developed for illustrating how the shape of the beam evolves as it propagates through the optical system. In Section 3, as a first practical use of that tool, we will show where it is possible to perform a measure of the wavefront and we will demonstrate the practical situations where the measurement using a SH is nonsense. In Section 4 we will concentrate on how one set of Zs (which define a wavefront at the entrance pupil of the setup) changes as the beam propagates up to an exit plane. We will illustrate two situations, corresponding to a highly convergent beam and to a nearly collimated beam. Finally, Section 5 will summarize the conclusions of our work.

*sbosch@ub.edu 
Rays begin to cross themselves at the bottom of surface 4; clearly cross in surface 5; in surfaces 6 and 7 the crossings are very involved in shape; in surfaces 8 and 9 rays do not cross. We will see later that when rays cross the wavefronts have not a simple one sheet shape, but complicated multi folded geometries.

The corresponding ZEMAX meridian plane scheme is presented in Figure2, illustrating the position of surfaces $4, \ldots 9$.

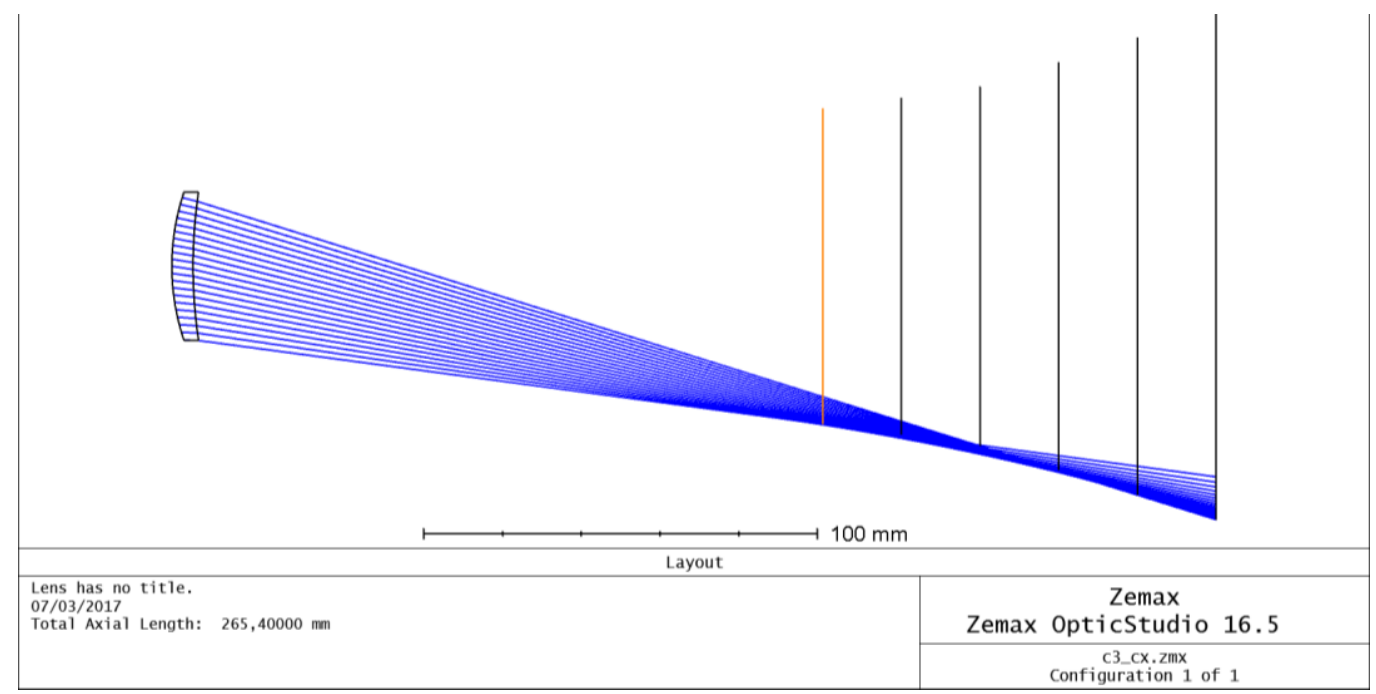

Figure 2. Meridian plane diagram showing the positions of the lens and surfaces $4, \ldots 9$ (the vertical lines, from left to right).

Figure 3 shows the 'contour plots' of the optical path of rays arriving from the object point to surfaces $4, \ldots 9$. For better clarity, we have substacted the accumulated optical path of the principal ray at the exit of the lens (which is 1342.4525 $\mathrm{mm}$ ). Of course, in zones where rays cross according to the previous spot diagrams, the contour plot is meaningless, since more than one ray (with different accumulated path) arrives ath the same point.
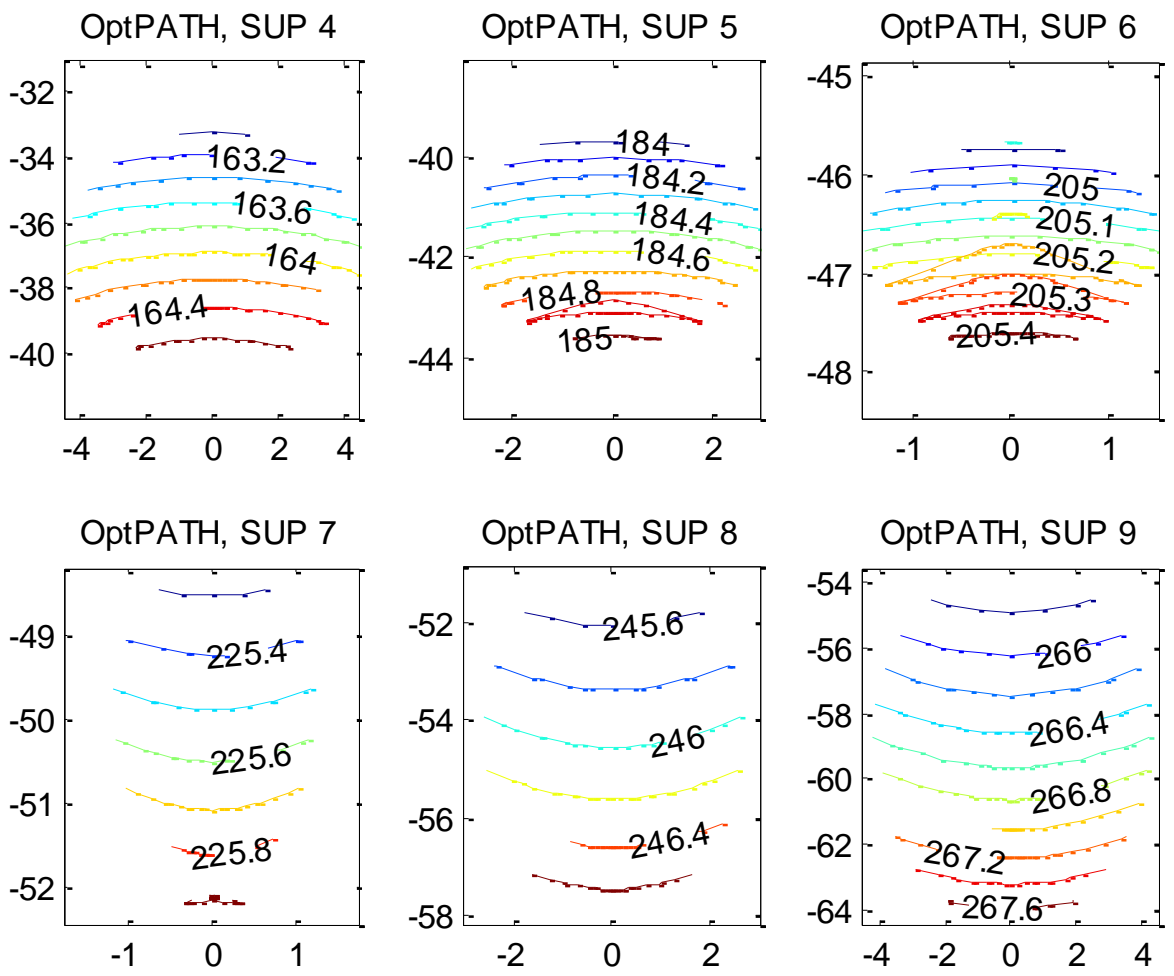

Figure 3. Contour plots of the optical path of rays arriving to surfaces $4, \ldots 9$. All units are $\mathrm{mm}$. 
The visualization of the wavefronts in 3D is more difficult. Thus we will do it first for meridional rays only. Figure 4 are the plots of the wavefronts near surfaces $4, \ldots 9$ as before. Abcissas are the $\mathrm{Z}$ coordinates (on the optical axis) and ordinates the Y-heigths. This kind of figures (for meridional rays only) have been explained in more detail when used in relation with caustics in our previous works. ${ }^{8-10}$
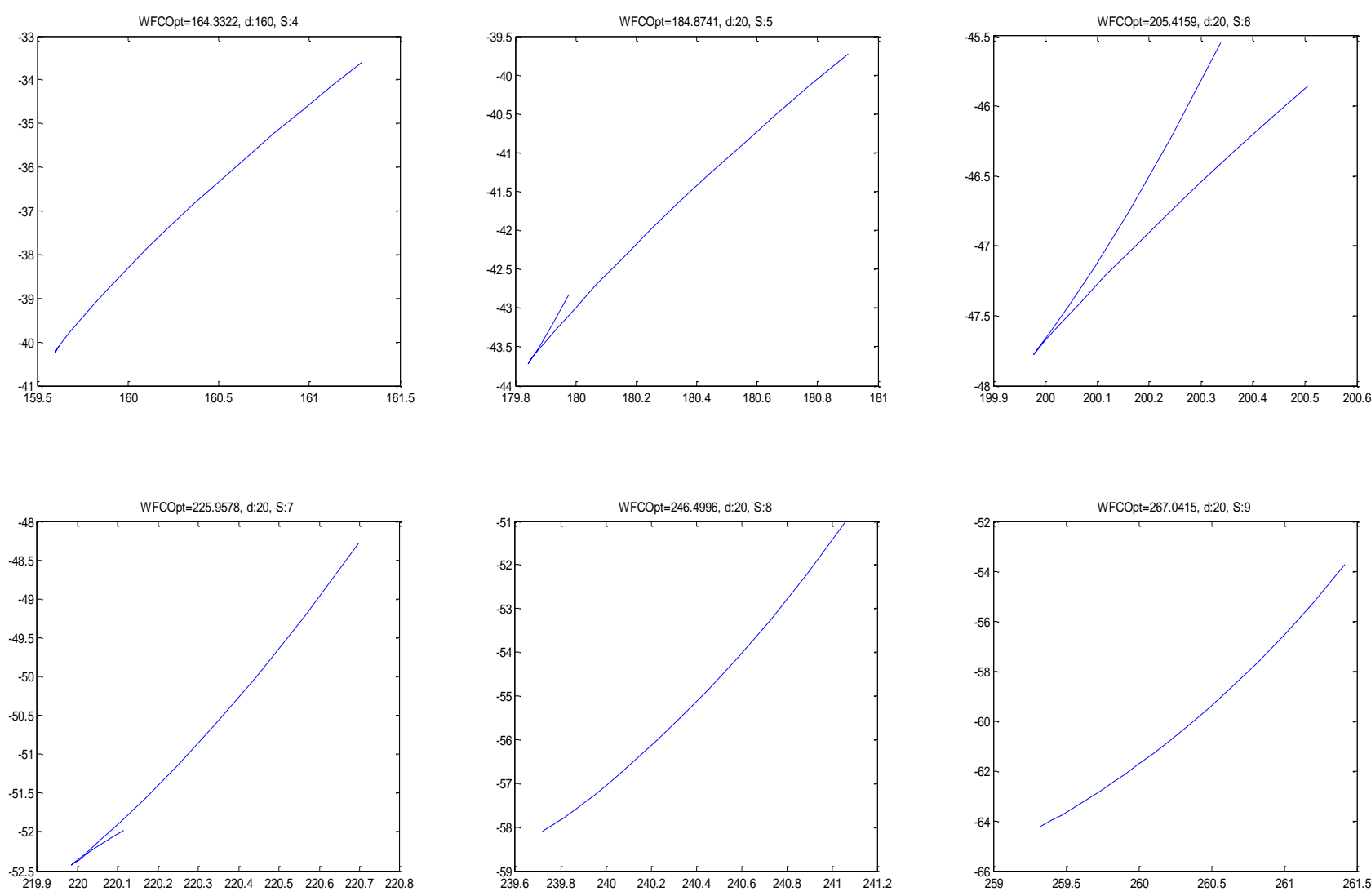

Figure 4. Meridional wavefront plots corresponding to the optical path of the chief ray arriving to surfaces $4, \ldots 9$.

In Figure 4, the top left subplot is the meridional cut of the wavefront which corresponds to an optical path of 164.3322 $\mathrm{mm}$ (above the accumulated optical path of the principal ray at the exit of the lens, which is $1342.4525 \mathrm{~mm}$ ). The shape shows a slight concavity towards the image side of our setup. The top central figure is the meridional cut of the wavefront which corresponds to an optical path of $184.8741 \mathrm{~mm}$ (above the accumulated optical path of the principal ray at the exit of the lens, as before). The shape shows the cut of the two sheets of the actual wavefront; the long right line corresponds to rays coming from the top side of the lens and the short left line to rays coming from the bottom side of the lens. Similarly, the top right figure is the meridional cut of the wavefront which corresponds to an optical path of $205.4559 \mathrm{~mm}$. The shape shows the cut of the two sheets of the wavefront; the right line corresponds to rays coming from the top side of the lens and the left line corresponds to rays coming from the bottom side of the lens.

To further clarify the origin of these two sheets, Figure 5 illustrates the cut of the meridional rays with surface 6 in our setup. In fact, the figure is only an enlarged portion of our previous Figure 2: the cut of surface 6 (the third one from the left there) and the meridional rays.

The same kind of comments are valid for bottom left subplot of Figure 4. The shape of the wavefront now is mostly convex to the image side of the setup and again shows the cut of the two sheets of the wavefront. Similarly, for the bottom central and right subplots of Figure4, the shapes now are simply convex to the image side of the setup (showing the divergent character of the wave). Note that the Gaussian image point (not shown in the figures) is at coordinates $\mathrm{z}=237.2637 \mathrm{~mm}, \mathrm{y}=-56.3562 \mathrm{~mm}, \mathrm{x}=0.0$.

The 3-D reresentations of the wavefronts are shown in Figure 6. The vertical axis is always the Y axis; the horizontal sagittal one is the $\mathrm{X}$ axis and the horizontal direction coincident with the optical axis of the setup is the $\mathrm{Z}$ axis. 


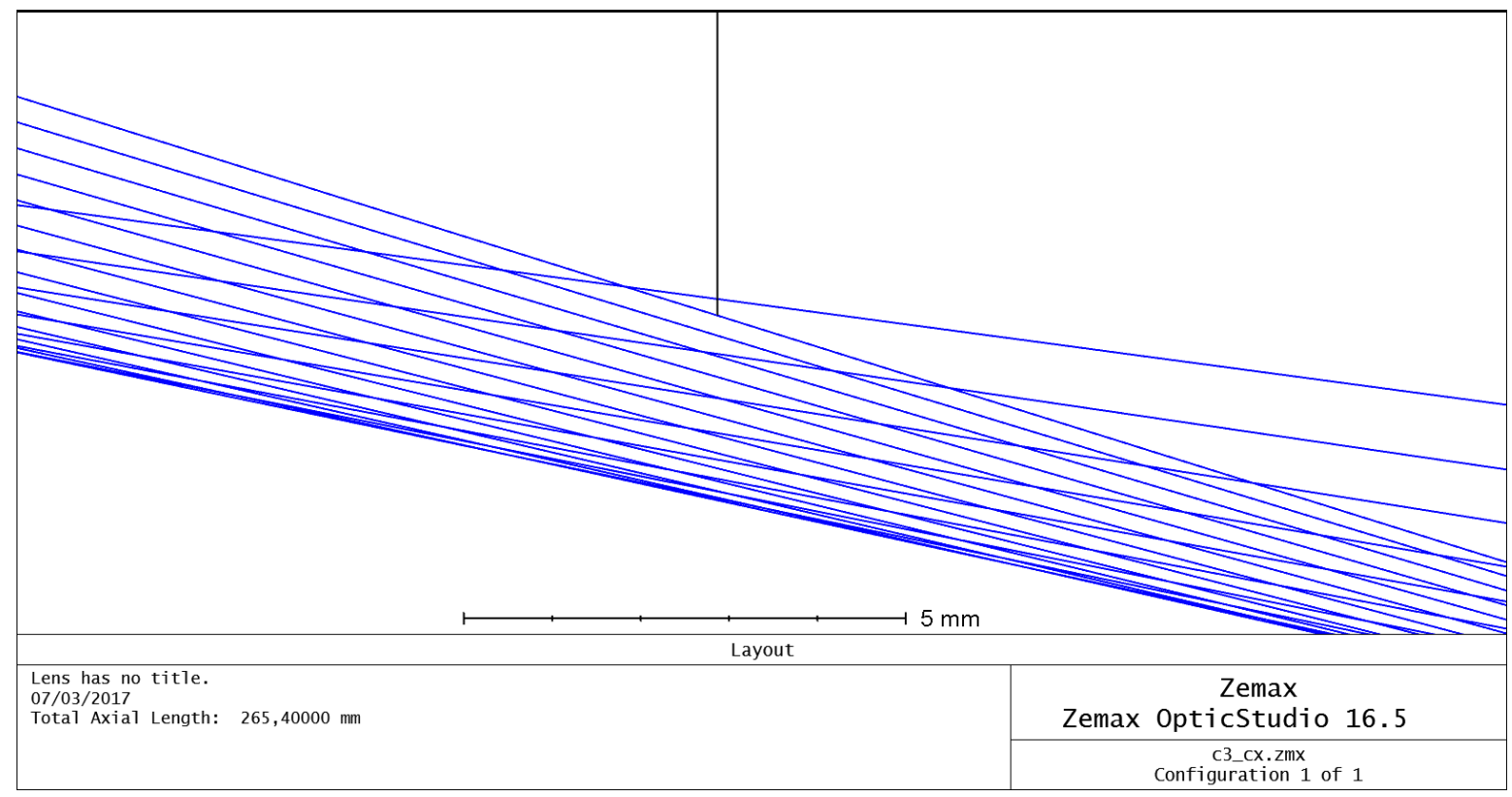

Figure 5. Cut of the meridional rays with surface 6 in our setup.
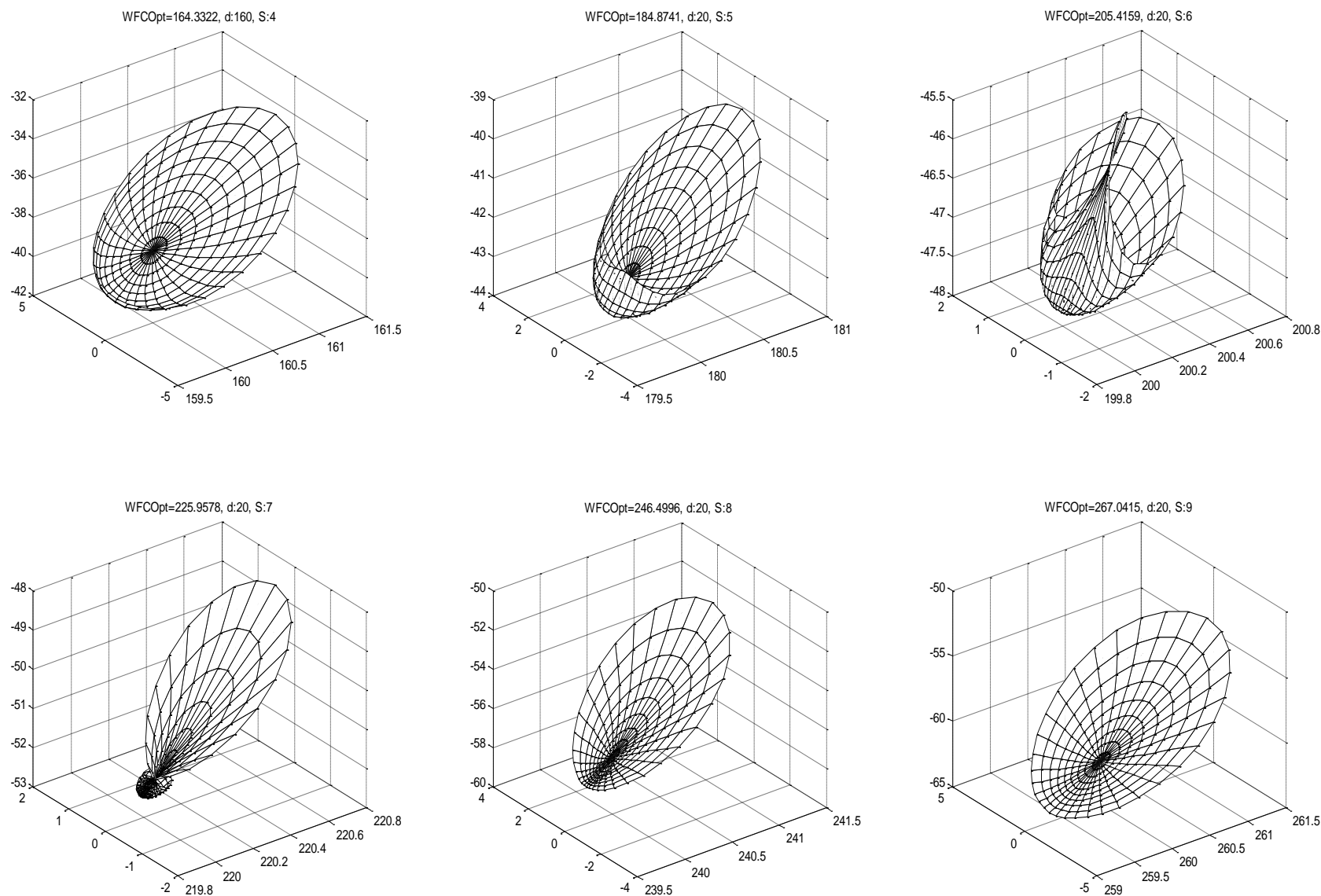

Figure $6.3 \mathrm{D}$ wavefronts corresponding to the optical path of the chief ray at the surfaces $4, \ldots 9$ in our setup. 


\section{WHERE IS IT POSSIBLE TO MEASURE WITH A SH SENSOR?}

Let us imagine we place a SH sensor centered at a point $\left(\mathrm{x}_{0}, \mathrm{y}_{0}, \mathrm{z}_{0}\right)$, always oriented normal to the optical axis. We will assume that the measuring area is square and that contains a regular array of microlenses. To be concise, let us take $11 \times 11$ microlenses, covering a 1x1 mm square area. The SH will sample the incoming beam and the subsequent analysis of the measurement will be made in terms of the spots focused by each individual microlens, as a local measure of the slope of the wavefront of the beam. ${ }^{3,4}$ The remaining relevant quantity is the focal length $\mathrm{f}$ of the microlenses. For our illustration, the space between microlenses is $1 /(11-1)=0.1 \mathrm{~mm}=100$ micron. We have taken $\mathrm{f}=6 \mathrm{~mm}$. For our computations we will assume that the spot for each microlens corresponds to the direction of the ray passing trough the center of the microlens. Since no ray of those initially traced will pass exactly trough the center of any microlens, an interpolation procedure is needed. We have used the function 'TriscatteredInterp' from MATLAB for that purpose.

Figure 7 illustrates where the $11 \times 11$ microlenses (dots) would focus the light when the $\mathrm{SH}$ is centered at the point $\left(\mathrm{x}_{0}=-\right.$ 1.5033, $\left.\mathrm{y}_{0}=-41.4775\right)$ in surface 5 . The spots are represented by crosses. One may note that the beam is basically convergent (the zone of crosses is smaller than the zone of dots) and pointing towards the bottom right side. Figure 8 is the same representation for the $\mathrm{SH}$ is centered at the point $\left(\mathrm{x}_{0}=1.0251, \mathrm{y}_{0}=-54.9998\right)$ in surface 8 . One may note that the beam diverges (the zone of crosses gets bigger than dots) and again aims towards the bottom right direction.

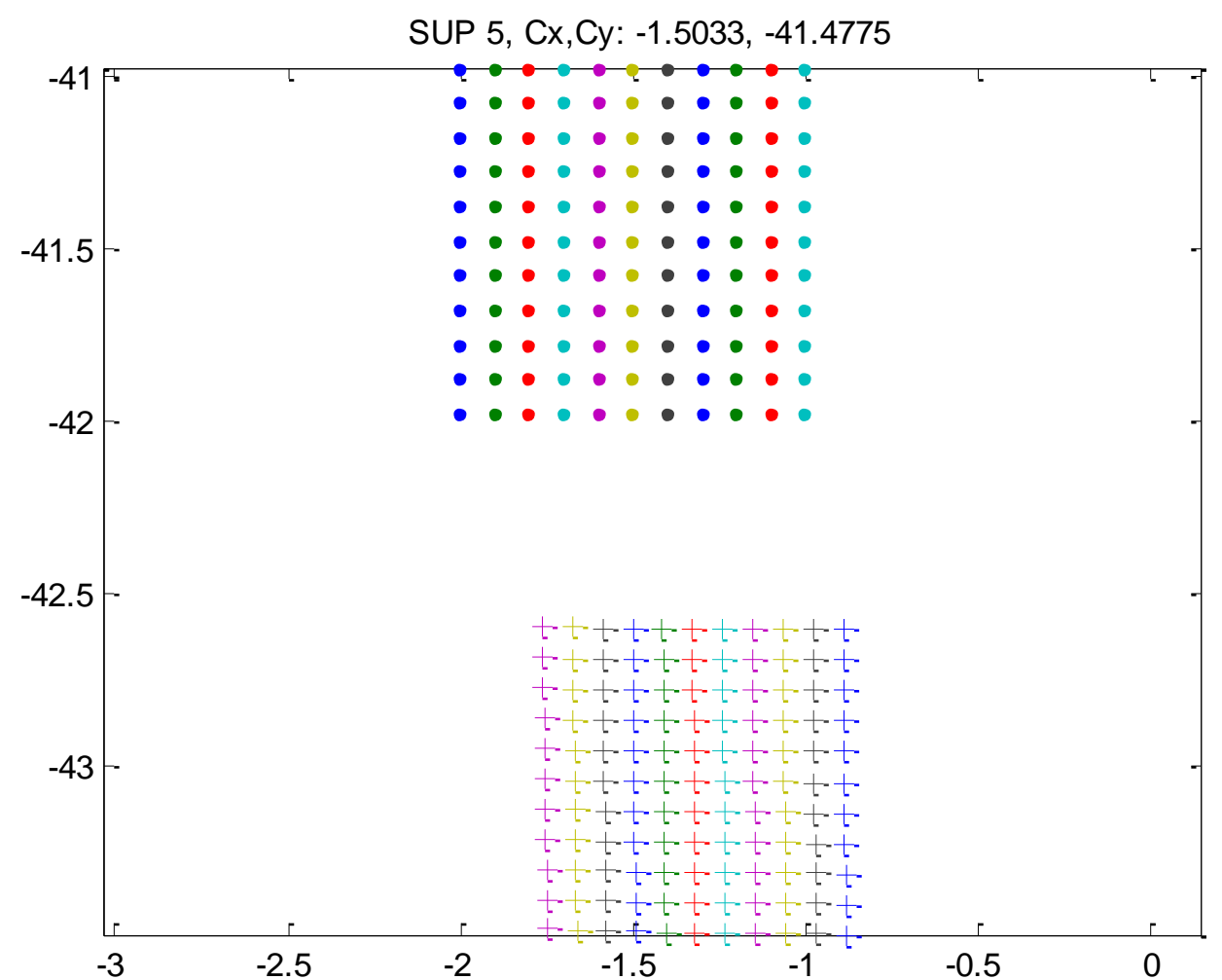

Figure 7. Center of the microlenses (dots) and corresponding spots (crosses) when the SH is centered in position point $\left(\mathrm{x}_{0}=-\right.$ $\left.1.5033, \mathrm{y}_{0}=-41.4775\right)$ in surface 5 .

The two cases just mentioned can be handled with the standard procedures for processing numerically the measured slopes in SH practical environment, leading to a 'zonal' reconstruction and eventually to a 'modal' reconstruction. ${ }^{2}$ This is possible because the central points of the SH sensor have been selected in zones where the wavefront was 'smooth enough', which is equivalent to say that 'no rays were crossing there'. This fact can be inferred by looking at the figure of the rings of spots and identifying the number of the surface and the position of the center of the SH. For comparison, the next Figure illustrates where the microlenses would focus the spots when the SH is centered at the point $\left(\mathrm{x}_{0}=-0.70053\right.$, $\mathrm{y}_{0}=-46.593$ ) in surface 6 . One may note that where rays cross themselves, the spots are, in fact, not well defined, as different parts of the light reaching a single microlens will aim at different directions . 
SUP 8, Cx,Cy: 1.0251, -54.9998

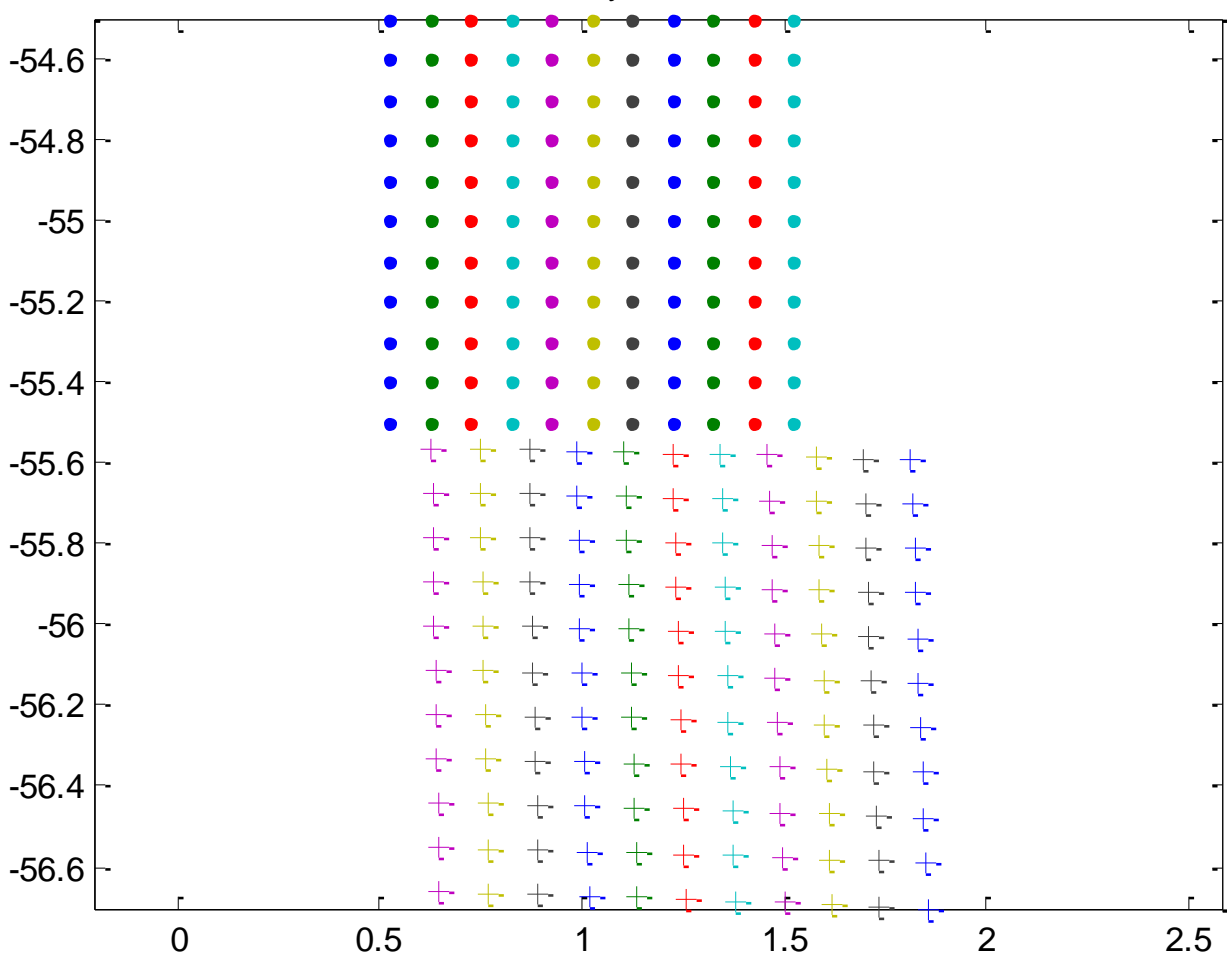

Figure 8. Microlenses (dots) and spots (crosses) when the SH is centered in position $\left(\mathrm{x}_{0}=1.0251, \mathrm{y}_{0}=-54.9998\right)$ in surface 8 .

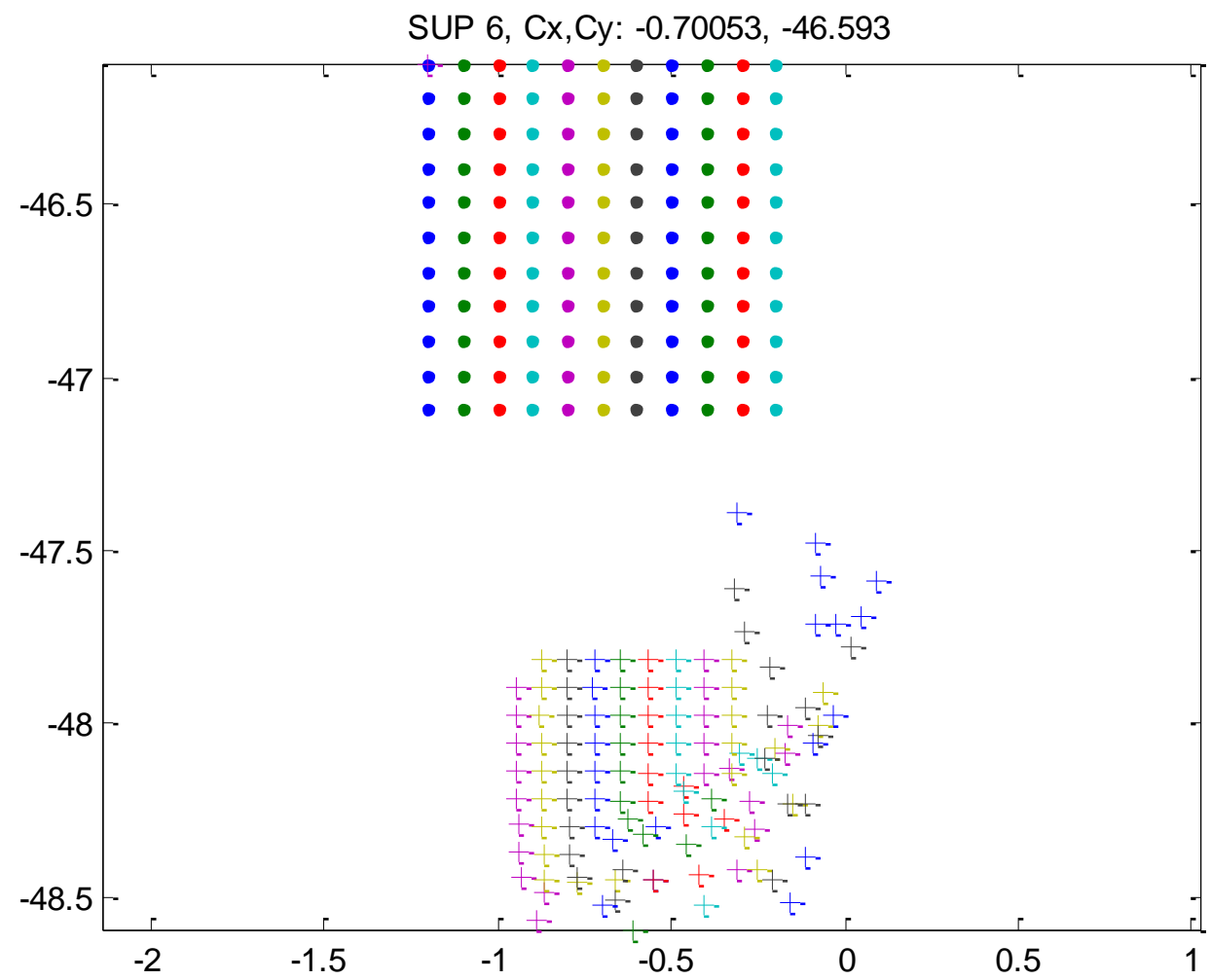

Figure 9. Microlenses (dots) and spots (crosses) when the SH is centered in position $\left(\mathrm{x}_{0}=-0.70053, \mathrm{y}_{0}=-46.593\right)$ in surface 6 . 


\section{HOW DO ZERNIKE COEFFICIENTS CHANGE AS THE BEAM PROPAGATES?}

Since we want to investigate the use of a SH sensor for the characterization of light beams in optical setups, we have added other capabilities to our MATLAB numerical tool. Assume a perfect spherical diverging wave arriving to the EnP of our setup. For conciseness, suppose this EnP is located just at the front (convex) side of the lens, as in the previous example. Assume that, at this plane, there is a phase wave plate that can be defined by a known set of Zs. As light crosses this plate and continues propagating through the setup, the shape of the wavefronts will evolve. Placing the $\mathrm{SH}$ sensor normally to the optical axis, centered at a certain transversal position, we will always sample a patch of the wavefront, as it has been assumed previously in this work. We show the results for two configurations of the above lens: 1) when it is generating a nearly collimated beam, or 2) when generating a highly convergent beam.

\subsection{Nearly collimated beam}

Using the same kind of scheme for the variables of the MATLAB script as in previous Section 2, now the point object is on axis at $230 \mathrm{~mm}$ of the convex side of the lens, originating a quite parallel beam after the lens.

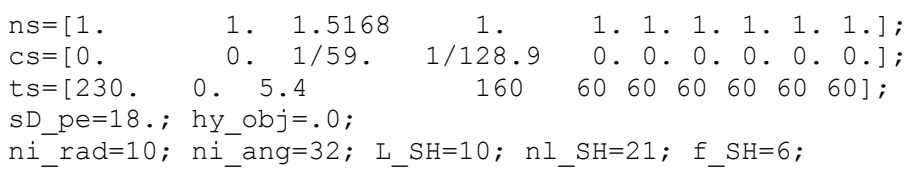

Using up to 13 Zs coefficients, we assume that their values at the EnP of the setup are given as follows:

nZks=13; Zks=zeros $(1, \mathrm{nZks})$;

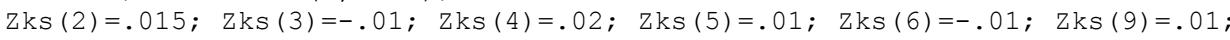

Now, for the spot diagrams (equivalent to Figure 1) we obtain Figure 10.
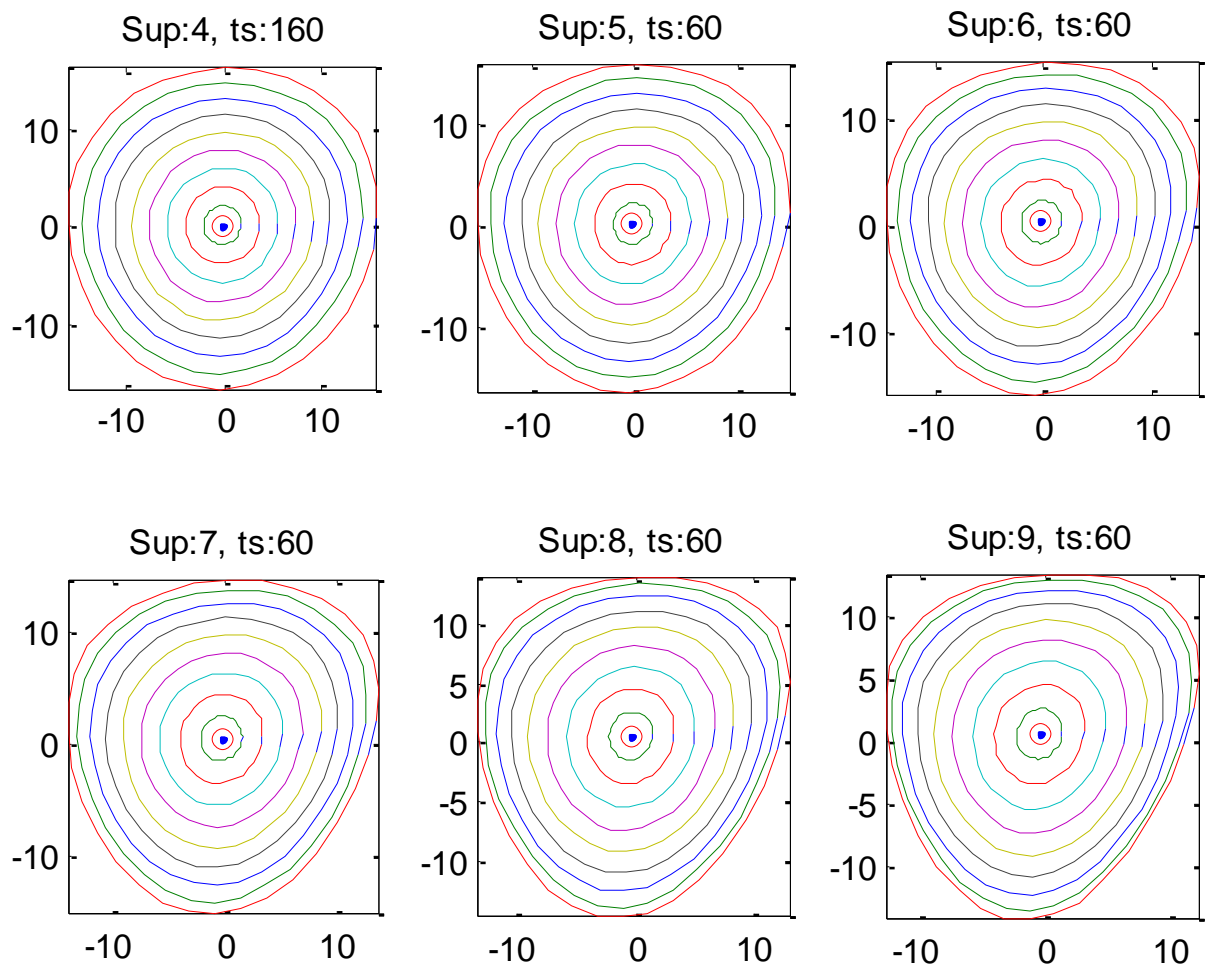

Figure 10. Spot diagrams corresponding to surfaces $4, \ldots 9$, as in Fig 1 . The EnP contains a phase plate defined by a set of Zs. Now the distance between planes is $60 \mathrm{~mm}$. 
For the present calculations, we take $10 \mathrm{~mm}$ for the side length of the SH sensor ( $\mathrm{L}_{-} \mathrm{SH}=10$;). We have calculated the Zs for the SH centered on the axis at surfaces 4, 5.. 9. The results are summarized in Figure 11. As expected, these six sets of results are quite similar in all cases.

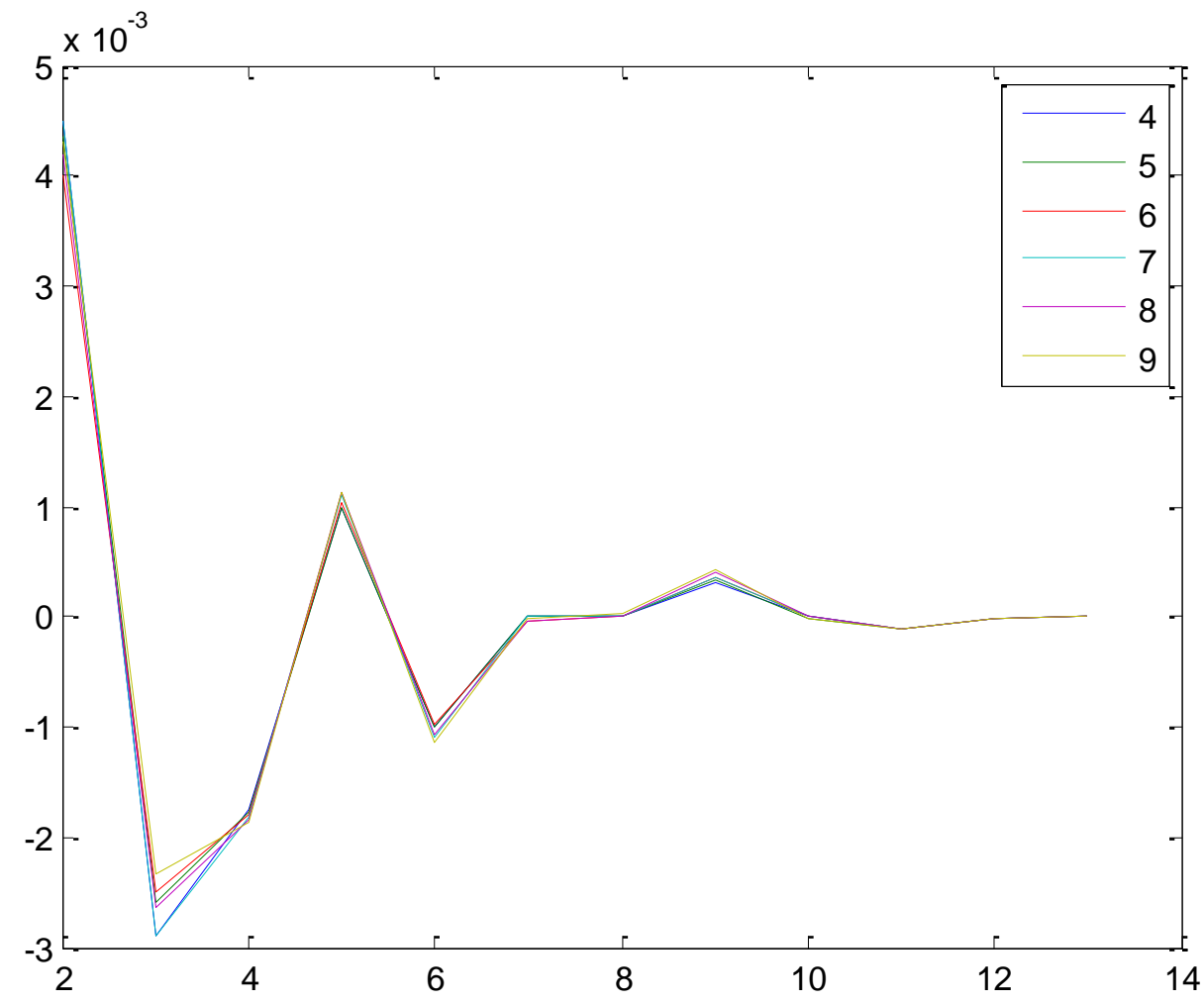

Figure 11. Values of the Zs on surfaces 4, $5 \ldots 9$ (5 mm semidiameter).

\subsection{Highly convergent beam}

With the same setup as above, assume that the only difference is a point source now placed on axis at $1300 \mathrm{~mm}$ from the convex side of the lens. Besides, we will take $2 \mathrm{~mm}$ for the size of the $\mathrm{SH}$ sensing area $\left(\mathrm{L}_{-} \mathrm{SH}=2\right.$; )

Figure 12 shows the spot diagrams at the planes 4, 5, 6, 7, 8 and 9. Clearly, surfaces 7 and 8 are not suitable for measuring using the SH. In any case, the shape of the beam changes dramatically; thus, the Zs will change abruptly between different planes. For this size of the beam, the Zs for the SH centered on the axis at surfaces 5, 6 and 9 are:

\begin{tabular}{|l|l|l|l|l|l|l|l|l|l|l|l|l|}
\hline & $\mathrm{Zs}(2)$ & $\mathrm{Zs}(3)$ & $\mathrm{Zs}(4)$ & $\mathrm{Zs}(5)$ & $\mathrm{Zs}(6)$ & $\mathrm{Zs}(7)$ & $\mathrm{Zs}(8)$ & $\mathrm{Zs}(9)$ & $\mathrm{Zs}(10)$ & $\mathrm{Zs}(11)$ & $\mathrm{Zs}(12)$ & $\mathrm{Zs}(13)$ \\
\hline EnP $(18 \mathrm{~mm}$ radius $)$ & 0.005 & -0.001 & 0.002 & -0.003 & -0.01 & 0. & 0. & 0.01 & 0. & 0. & 0. & 0. \\
\hline surf 5 $(1 \mathrm{~mm}$ rad.) & $-0,0004$ & 0,0001 & $-0,0047$ & $-0,0002$ & $-0,0005$ & 0. & 0. & 0.0001 & 0. & 0. & 0. & 0. \\
\hline surf 6 $(1 \mathrm{~mm}$ rad.) & 0,0002 & 0,0009 & $-0,0073$ & $-0,0005$ & $-0,0014$ & 0. & 0. & 0.0005 & 0. & 0.0001 & 0. & 0. \\
\hline surf 9 $(1 \mathrm{~mm}$ rad. $)$ & $-0,0003$ & 0,0006 & 0,0012 & $-0,001$ & $-0,0033$ & 0.0001 & 0. & -0.0018 & 0. & -0.0002 & 0.0001 & 0.0002 \\
\hline
\end{tabular}

Anyway, it is important to note that our numerical procedures, by following track of the rays and optical paths along the setup, are able to calculate the corresponding values of the Zs. 

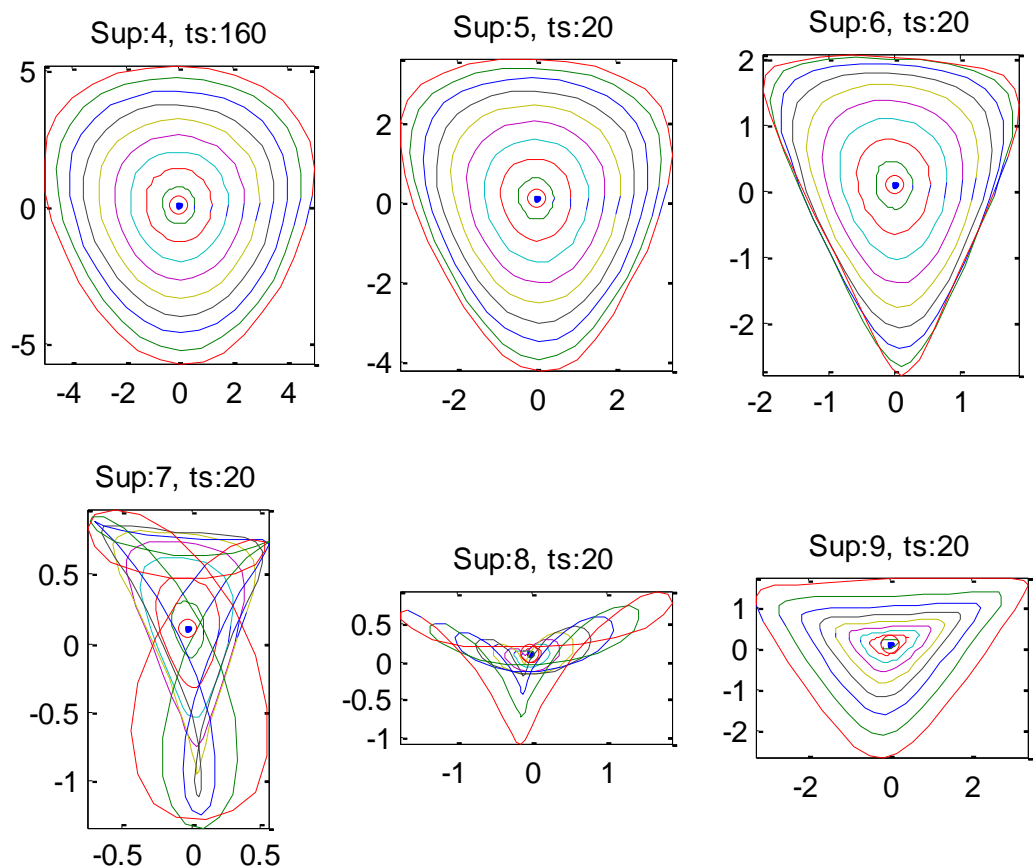

Figure 12. Spot diagrams corresponding to surfaces $4, \ldots 9$, when the poin source is on axis at $1300 \mathrm{~mm}$ and the EnP contains a phase plate defined by a set of Zs. Now the distance between planes is $20 \mathrm{~mm}$.

\section{CONCLUSIONS}

The main goal of this work is the development of a numerical tool that allows us to evaluate the propagation of an optical beam through an optical system, showing the beam and wavefront shape at different planes. Thanks to this tool we were able to show the evolution of the wavefront and the presence of planes where the optical path is bivaluated. This kind of information may be important when pupil conjugation is performed by using just one lens, or after passing through a beam diameter compressor built with a Galilean telescope, for example. Additionally the numerical tool allowed us to study how the modal coefficients of the wavefront Zernike expansion change as the beam propagates. Finally we expect that the present numerical tool will also help in the determination of a transformation rule for the calculation of the modal coefficients of a Zernike expansion at one plane knowing the coefficients at another plane.

\section{ACKNOWLEDGEMENTS}

The authors acknowledge the 'Agencia Estatal de Investigación' (AEI) and the 'Fondo Europeo de Desarrollo Regional' (FEDER), under project FIS2016-77319-C2-2-R of the Spanish 'Ministerio de Economia, Industria y Competitividad'.

\section{REFERENCES}

[1] W.T. Welford, Aberrations of Optical Systems, Adam Hilger, Bristol (1986).

[2] V.N. Mahajan, Optical Imaging and Aberrations, (SPIE Press, Bellingham, 1998)

[3] HASO software user guide. Imagine Optic, 2006.

[4] http://fp.optics.arizona.edu/icwvant/Zernikes/ZernikePolynomials.htm

[5] MATLAB, The MathWorks Inc., Natick, MA, 2000

[6] ZEMAX Development Corporation, 3001 112th Avenue NE, Suite 202, Bellevue, WA 98004-8017 USA

[7] W.J. Smith, Modern Optical Engineering (McGraw-Hill, New York, 2000)

[8] S. Bosch, "Ray tracing and scalar diffraction calculations of wavefronts, caustics and complex amplitudes in optical systems," J. Mod. Opt. 58, 146-153 (2011)

[9] J. Ferré-Borrull, and S. Bosch, "Formal description of diffraction in optical systems: calculations and experimental evidence," Appl. Phys. Lett. 85, 2718-2720 (2004)

[10] S. Bosch, and J. Ferré-Borrull, “The focal shift in converging of waves" J. Mod. Opt. 50, 2221-2229 (2004) 